\title{
Comment on Calculation of Positron Flux from Galactic Dark Matter
}

\section{Bibhushan SHAKYA*}

Laboratory of Elementary Particle Physics, Cornell University, Ithaca, New York 14853, USA

E-mail: bs475@cornell. edu

\section{Maxim PERELSTEIN}

Laboratory of Elementary Particle Physics, Cornell University, Ithaca, New York 14853, USA

E-mail: mp325ecornell.edu

\begin{abstract}
Energetic positrons can serve as an important indirect signature of dark matter. Computing the positron flux expected in a given dark matter model involves solving transport equations in the galaxy. Existing calculations solve the equations inside the diffusion zone, where galactic magnetic fields confine positrons, and assume vanishing positron density on the boundaries of this zone. However, in many models, a substantial fraction of the dark matter halo lies outside the diffusion zone, and positrons produced there can contribute to the flux at Earth. We calculate this correction for a variety of models. The corresponding enhancement in the flux of energetic photons produced by the inverse Compton scattering of positrons on starlight and cosmic microwave background is also calculated. Maximal flux enhancements of order $20 \%$ are found in both cases.
\end{abstract}

Identification of Dark Matter 2010

July 26 - 302010

University of Montpellier 2, Montpellier, France

\footnotetext{
${ }^{*}$ Speaker.
} 


\section{Introduction and Formalisms}

Proper calculation of positron density from dark matter annihilation in the Milky Way galaxy is crucial for fits to possible signals of dark matter, such as the recent PAMELA [1] (positron fraction) and Fermi [2](combined $e^{+} e^{-}$) anomalies. Positrons have complicated interactions with the interstellar medium; approximate, semi-analytical solutions for positron density in the galaxy are obtained by solving the steady state transport equation (see e.g. Refs. [3, 4]):

$$
-\nabla[K(\mathbf{x}, E) \nabla \psi]-\frac{\partial}{\partial E}[b(E) \psi]=q(\mathbf{x}, E),
$$

where $\psi(\mathbf{x}, E, t)=d n_{e^{+}} / d E$ is the positron density per unit volume per unit energy, $K$ is the diffusion coefficient describing the interaction of the positrons with the stochastic galactic magnetic field, $b$ is the rate at which positrons lose energy due to synchrotron emission and inverse Compton scattering (ICS), and $q$ is the source term, in our case due to dark matter annihilation or decay.

In conventional solutions, the positron density is obtained by solving the transport equation in a thin cylinder (half-thickness $L$, radius $R=20 \mathrm{kpc}$ ), the diffusion zone, that contains most of the galactic magnetic fields that trap positrons. Contributions from outside this region are ignored under the assumption that positrons produced here propagate freely and escape. Assuming a position independent diffusion coefficient $K(E)=K_{0}(E / G e V)^{\delta}$, expanding the positron density in Bessel and Fourier series, and taking the Bessel and Fourier transforms of the transport equation, the following solution is obtained [4]:

$$
\begin{gathered}
\psi(r, z, \varepsilon)=\frac{\tau_{E}}{\varepsilon^{2}} \int_{\varepsilon}^{\varepsilon_{\max }} d \varepsilon_{S} f\left(\varepsilon_{S}\right) \sum_{i} \sum_{n} J_{0}\left(\frac{\alpha_{i} r}{R}\right) \sin \left(\frac{n \pi(z+L)}{2 L}\right) \exp \left[-\omega_{i, n}\left(t-t_{S}\right)\right] R_{i, n}, \\
\omega_{i, n}=K_{0}\left[\left(\frac{\alpha_{i}}{R}\right)^{2}+\left(\frac{n \pi}{2 L}\right)^{2}\right],
\end{gathered}
$$

$R_{i, n}$ are coefficients of the Bessel-Fourier expansion of the source term, and $\alpha_{i}$ are the zeros of $J_{0}$; the reader is referred to [5] for more details.

The dark matter halo of the galaxy, however, extends significantly beyond this region; for instance, for $L=1 \mathrm{kpc}$ and dark matter with an isothermal profile, the diffusion zone contains only about $10 \%$ of the dark matter mass. Positrons produced in the halo outside the diffusion zone can enter it and contribute to the positron density there. The flux calculations should therefore include this contribution. We accomplish this by solving Eq. (1.1) in a larger cylinder extending out to $|z|=D$ - with $D>L$ such that essentially all of the dark matter is contained within this cylinder - and imposing the boundary condition $\psi=0$ at $z= \pm D$. The diffusion coefficient remains $K(E)=K_{0}(E / G e V)^{\delta}$ for $|z| \leq L$ but increases smoothly to $K(E)=K_{1}(E / G e V)^{\delta}$, with $K_{0} \ll K_{1}$, for $L+d \leq|z| \leq D$ over the small interval $d \ll L$ [5]. The transport equation can then be solved in a manner analogous to the conventional solution to obtain a similar solution [5]

$$
\psi(r, z, \varepsilon)=\frac{\tau_{E}}{\varepsilon^{2}} \int_{\varepsilon}^{\varepsilon_{\max }} d \varepsilon_{S} f\left(\varepsilon_{S}\right) \sum_{i} \sum_{n} J_{0}\left(\frac{\alpha_{i} r}{R}\right) \sin \left(\frac{n \pi(z+D)}{2 D}\right)\left(\exp \left[-\left(t-t_{S}\right) \mathbf{A}_{i}\right] \mathbf{R}_{i}\right)_{n}
$$

The non-diagonal matrices $\mathbf{A}_{i}$, which are analogous to $\omega_{i, n}$ in Eq. (1.3), are a consequence of the position dependent diffusion coefficient, which couples different Fourier modes; we refer to [5] for more details. The exponential in Eq. (1.4) can be calculated, for instance, by working in a basis where $\mathbf{A}_{i}$ is diagonal. 


\section{Results: Positron Fluxes}

The positron densities obtained from Eq. (1.2) and Eq. (1.4) can be compared for different dark matter distribution profiles, propagation models, and annihilation and decay channels. Following the leptophilic models invoked to explain the PAMELA and Fermi results, we considered annihilation into (i) $e^{+} e^{-}$, (ii) $\mu^{+} \mu^{-}$, (iii) $4 \mathrm{e}$, and (iv) $4 \mu$; the latter two channels are assumed to result from a pair of intermediate particles $\phi$ that dark matter first annihilates into ${ }^{1}$. MED and M2 models, which use $L=4 \mathrm{kpc}$ and $L=1 \mathrm{kpc}$ respectively, were used for galactic propagation, and Moore, NFW, and cored isothermal profiles were employed for the dark matter distribution (details for all these profiles and propagation models are presented in [5]). Figure 1 shows some of the resulting enhancements in computed positron flux at Earth from using Eq. (1.4) instead of Eq. (1.2) for $3 \mathrm{TeV}$ annihilating dark matter $\left(m_{\phi}=1 \mathrm{GeV}\right.$ where applicable).
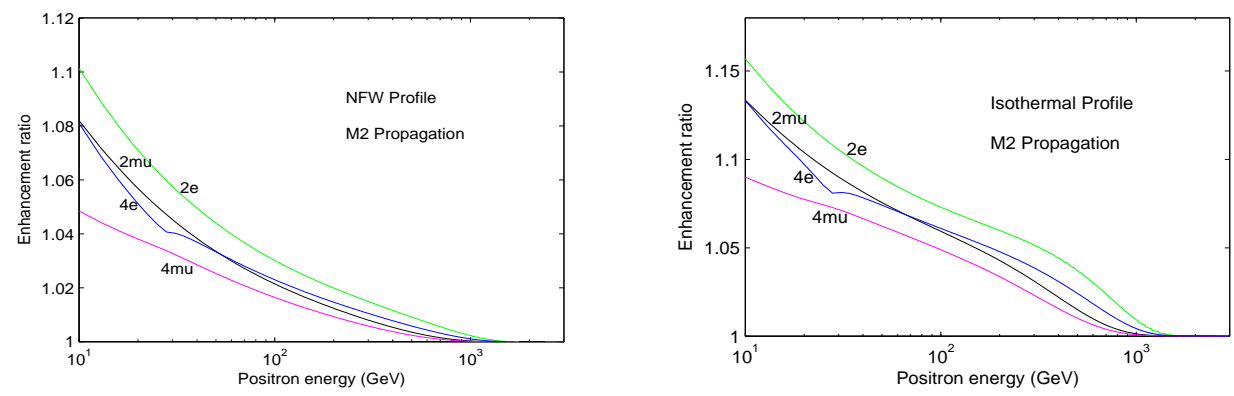

Figure 1: Positron flux enhancement for annihilating dark matter, for $m_{\chi}=3 \mathrm{TeV}$.

The results show many expected features. The enhancement is largest for annihilation into $e^{+} e^{-}$, which has the most energetic input spectrum, and progressively decreases for less energetic input spectra. The plots are consistent with the notion that for $\mathrm{M} 2$ propagation the spectrum at Earth is influenced largely by sources within a few kpc and, in particular, for the flux measured in the solar neighborhood, is insensitive to the large cusp at the center of the galaxy. The enhancement is larger at lower positron energies: positrons lose energy as they propagate through the intergalactic medium and hence energetic positrons entering from the halo outside the diffusion zone arrive at Earth at lower energies. The enhancement is found to be negligible (percent level) for MED propagation: there is negligible amount of dark matter outside the diffusion zone compared to inside it in this case, and the diffusion zone boundary is also farther away from the Earth. In the diffusion zone, the corrections are greatest for regions close to the diffusion zone boundary, and become progressively smaller towards the galactic plane.

Larger corrections are expected for decaying dark matter scenarios since the dark matter annihilation rate is proportional to $\rho_{\chi}^{2}$ - where $\rho_{\chi}$ stands for the dark matter density — but the decay rate is proportional to $\rho_{\chi}$, hence a larger fraction of positrons get produced outside the diffusion zone. Our results show that for M2 propagation the corrections to positron flux for decaying dark matter are only a few percent greater than those in the annihilating scenarios, presumably because the region of influence only extends to a few kpc, where spatial variations in $\rho_{\chi}^{2}$ and $\rho_{\chi}$ are similar.

\footnotetext{
${ }^{1}$ Such $\phi$ s are essential in the Sommerfeld enhancement mechanism used as a means to explain the enhanced cross sections needed for a dark matter interpretation of PAMELA and Fermi data [6].
} 

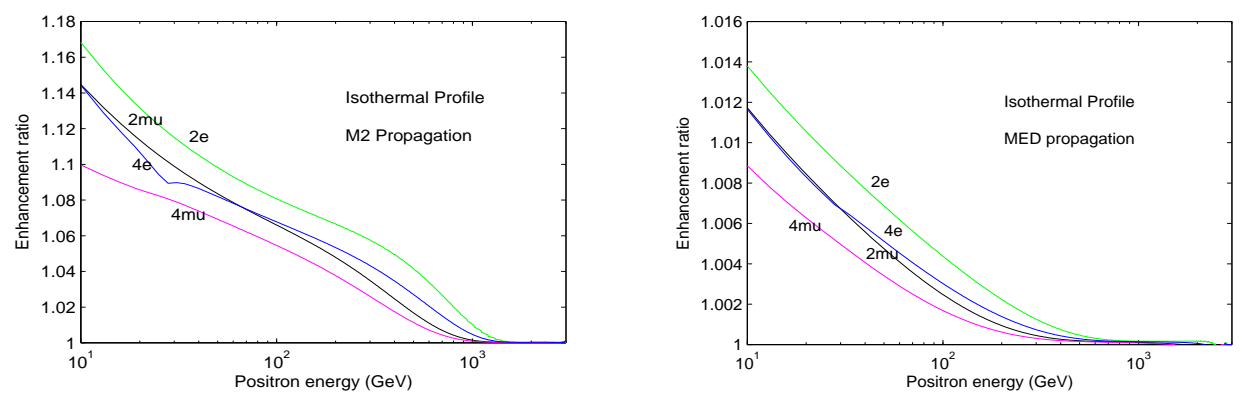

Figure 2: Positron flux enhancement for decaying dark matter, for $\mathrm{m}_{\chi}=6 \mathrm{TeV}$.

Corrections for MED propagation continue to remain negligible. Some sample plots are shown in Figure 2.

\section{Gamma Rays from Inverse Compton Scattering}

Observable positron flux corrections (above) are only sensitive to the positron density at the Earth's position, which lies at $z=0$ and receives the smallest corrections. The more significant corrections occur close to the diffusion zone boundary, and can be probed by observing gamma ray flux from inverse Compton scattering (ICS) since a photon scattering off an energetic positron towards the Earth from this boundary will travel unperturbed through the interstellar medium and can be detected at Earth. We used a semi-analytic formalism for calculation of ICS gamma ray flux, as outlined in [8], [9], and [5].
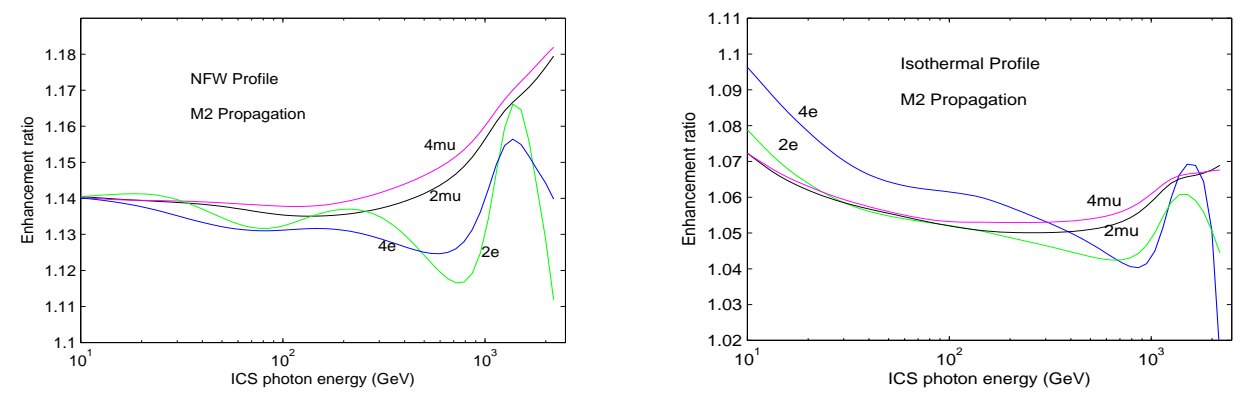

Figure 3: ICS gamma ray flux enhancement for annihilating dark matter, $m_{\chi}=3 \mathrm{TeV}$.

Figure 3 shows, for M2 propagation and annihilating dark matter, the enhancement in ICS gamma ray flux along a line of sight from the Earth to the point on the diffusion zone boundary closest to the galactic center. In this case, the largest contribution to the ICS flux enhancement comes from regions close to the diffusion zone boundary: the photon density is the greatest there because of the proximity to the galactic center, and the positron density is zero or close to zero in the conventional formalism because of the boundary conditions but can be significant in our extended formalism. In contrast to positrons, enhancement in ICS flux is seen to persist at all energies up to the dark matter mass since photons travel through the interstellar medium without losing energy. The three 'bumps' in the plots correspond to the three different galactic gamma-ray components: $\mathrm{CMB}$, starlight, and starlight re-scattered off dust. 


\section{Concluding Remarks}

Including contributions from the dark matter halo outside the diffusion zone to positron density coming from dark matter annihilation or decay can result in corrections of up to $10-15 \%$ in the observed positron flux at Earth and up to $20-25 \%$ in the ICS gamma ray flux. For positron flux, the enhancement is progressively lower at higher energies since positrons coming in from the halo lose energy in the diffusion zone and arrive at detectors with lowered energies. For ICS gamma ray flux, the bulk of the correction comes from photons scattering off energetic positrons close to the diffusion zone boundary, hence the enhancement is maintained even at the highest photon energies. The enhancements are most significant for the M2 propagation model, where the diffusion zone is only $2 \mathrm{kpc}$ thick and most of the dark matter halo lies outside this zone. For the MED model (and presumably other models with larger $L$ ), the corrections are negligible. At present, experimental uncertainties on flux measurements as well as astrophysical uncertainties in the positron and ICS fluxes remain significantly greater than the additional contribution calculated here; nevertheless, these contributions from outside the diffusion zone do need to be considered when accuracy to better than $\sim 25 \%$ is needed.

\section{Acknowledgments}

This research is supported by the U.S. National Science Foundation through grant PHY0757868 and CAREER award PHY-0844667.

\section{References}

[1] O. Adriani et al. [PAMELA Collaboration], Nature 458, 607 (2009) [arXiv:0810.4995 [astro-ph]].

[2] A. A. Abdo et al. [The Fermi LAT Collaboration], Phys. Rev. Lett. 102, 181101 (2009) [arXiv:0905.0025 [astro-ph.HE]].

[3] E. A. Baltz and J. Edsjo, Phys. Rev. D 59, 023511 (1998) [arXiv:astro-ph/9808243].

[4] T. Delahaye, R. Lineros, F. Donato, N. Fornengo and P. Salati, Phys. Rev. D 77, 063527 (2008) [arXiv:0712.2312 [astro-ph]].

[5] M. Perelstein and B. Shakya, Phys. Rev. D 82, 043505 (2010) [arXiv:1002.4588 [astro-ph.HE]].

[6] N. Arkani-Hamed, D. P. Finkbeiner, T. R. Slatyer and N. Weiner, Phys. Rev. D 79, 015014 (2009) [arXiv:0810.0713 [hep-ph]].

[7] D. Maurin, F. Donato, R. Taillet and P. Salati, Astrophys. J. 555, 585 (2001) [arXiv:astro-ph/0101231].

[8] P. Meade, M. Papucci, A. Strumia and T. Volansky, Nucl. Phys. B 831, 178 (2010) arXiv:0905.0480 [hep-ph].

[9] M. Cirelli and P. Panci, Nucl. Phys. B 821, 399 (2009) [arXiv:0904.3830 [astro-ph.CO]]. 